\title{
Automatic Diagnosis of Iron and steel Equipment using Infrared Thermography
}

\author{
by Y. Laib dit Leksir ${ }^{* * *}$, S. Bouhouche*, M. S. Bouchrit ${ }^{* *}$ \\ "Iron and Steel Applied Research Unit, BP 196, URASM- CSC, Annaba \\ **Process Control Laboratory, ENP, BP 182, 16200 El-Harrach, Algiers, yaziddl@yahoo.fr
}

\begin{abstract}
Among the applicability of infrared thermography we find the iron and steel industry, where the inspection and the maintenance of machines play a significant role in this field to avoid breakdowns and damages. The inspection using Infrared thermography technology can be conducted efficiently by keeping a distance from the inspected equipment. There is no need to halt equipment operation while an inspection is going on. Since the collection of information for inspection is contactless, hazardous operations can be avoided. The main information gained from this technology is in the form of thermal image. Any abnormal condition of an inspected object will be reflected as an abnormal spot. The objective of this work is to visualize the anomalies existing in iron and steel industry during an inspection of some machines using A40 Flir camera. Then, we threshold these images in order to show the zone of interest and we make comparison between a few thresholding methods of thermal images obtained to choose the best one.
\end{abstract}

\section{Introduction}

One area where IRT has played an important role is in the Iron and steel industry. This type of industry is categorized as a heavy industry with high investment cost, operational cost, and maintenance cost, along with high requirements for safety. Any problem found in the operating facilities must be detected early since breakdown of equipment will affect other equipment or even the entire operation of the plant. Maintenance should be scheduled properly and regularly because the shutdown or startup of equipment cannot be carried out abruptly as it is related to the operational cost and the overall system's performance.

The plant maintenance profits for a long time from the use of the thermal cameras. The decisive advantage of thermography is to allow the estimate of equipment's quality in normal exploitation, without stopping of the machines and before the effective failure. Of course, it is necessary that degradation leads to an evolution of the distributions of temperatures: what is very often the case. Thermography reveals what will arrive, if anything is not done.

Thermal image segmentation is a critical preprocess step in image analysis and pattern recognition [1-4]. Thresholding is one of the most important and effective techniques, and plays a key role when segmenting obtained images with distinctive gray levels corresponding to object and background. Its aim is to find an appropriate threshold for separating the object from the background. Thresholded result is a binary image where all pixels with gray levels higher than the determined threshold are classified as object and the rest of pixels are assigned to background, or vice versa. The technique can serve a variety of applications, such as biomedical image analysis [5], handwritten character identification [6], automatic target recognition [7] and change detection [8]. Thresholding methods can be classified into parametric and nonparametric approaches [9-17]. The former assumes gray level distribution of each class obey a given distribution, usually a normal distribution, and finds the optimal threshold by estimating the parameters of the distribution using the given histogram. This typically leads to a nonlinear estimation problem of expensive computation. The latter usually determines the optimal threshold by optimizing certain objective function, such as between-class variance [11], variance [13], entropy [14-16] and energy [17]. The non parametric approach is proved to be more robust and accurate than the parametric one.

The remainder of this paper is organized as follows: Section 2 introduces three thresholding methods. The performance of the studied methods is tested on a variety of images of steel and industry equipments and compared the results with other methods in Section 3. Conclusions appear in Section 4.

\section{Thresholding algorithm}

In this section, three thresholding methods, i.e., Otsu's, fuzzy c means clustering and LIW's methods, are first briefly reviewed, and also discussed.

\subsection{Histogram based thresholding}

\section{1. 1 Definition}

Let the pixels of the image be represented by $L$ gray levels $\{0,1,2, \ldots, L-1\}$. The number of pixels in level $i$ is denoted by $h i$ and the total number of pixels is denoted by $N$. To simplify, the gray level histogram is normalized and regarded as the estimation of probability distribution function

$$
p_{i}=h_{i} / N, p_{i} \geq 0, \sum_{i=0}^{L-1} p_{i}=1
$$

Suppose we divide the pixels into two classes $C_{0}$ and $C_{1}$ by a threshold value at $k . C_{0}$ and $C_{1}$ denote pixels with levels $[0,1, \ldots, k]$ and $[k+1, \ldots, L-1]$, respectively. The probabilities of class occurrences $\omega$, class mean levels $\mu$, and class variance for both classes is given by: 
$\omega_{0}=\sum_{i=0}^{k} p_{i} ; \omega_{1}=\sum_{i=k+1}^{L-1} p_{i}=1-\omega_{0} ;$

$\mu_{0}=\sum_{i=0}^{k} i p_{i} / \omega_{0} ; \mu_{1}=\sum_{i=k+1}^{L-1} i p_{i} / \omega_{1}=\frac{\mu_{T}-\mu_{k}}{1-\omega_{0}} ;$

$\sigma_{0}^{2}=\sum_{i=0}^{k}\left(i-\mu_{0}\right)^{2} p_{i} ; \sigma_{1}^{2}=\sum_{i=k+1}^{L-1}\left(i-\mu_{1}\right)^{2} p_{i} ;$

Where $\mu_{k} \sum_{i=0}^{k} i p_{i} ; \mu_{T}=\sum_{i=0}^{L-1} i p_{i} ; \sigma_{T}^{L-1}=\sum_{i=0}^{L-1}\left(i-\mu_{T}\right)^{2} p_{i}$

$\mu_{T}$ and $\sigma_{T}$ are respectively the total mean and standard deviation.

\subsubsection{Otsu's Variance Method}

Otsu [11] suggested minimizing the weighted sum of within-class variances of the object and background pixels to establish an optimum threshold. Recall that minimization of within-class variances is equivalent to maximization of between-class variance. To measure the thresholding performance, a criterion measure is introduced by Otsu:

$\eta=\sigma_{B}^{2} / \sigma_{T}^{2}$

Where $\sigma_{B}^{2}=\omega_{0}\left(\mu_{0}-\mu_{T}\right)^{2}+\omega_{1}\left(\mu_{1}-\mu_{T}\right)^{2}$

is the between-class variance which can be simplified to

$\sigma_{B}^{2}=\omega_{0} \omega_{1}\left(\mu_{1}-\mu_{2}\right)^{2}$

The optimal threshold $k_{\text {opt }}$ is given by maximizing $\eta$, or equivalently maximizing $\sigma_{B}^{2}$, since $\sigma_{T}^{2}$ is independent of $\mathrm{k}$.

$k_{\text {opt }}=\max _{k}\left\{\sigma_{B}^{2}\right\}$

\section{2 fuzzy c means clustering method}

The goal of a clustering analysis is to divide a given set of data or objects into a cluster, which represents subsets or a group [18]. The partition should have two properties:

1- Homogeneity inside clusters: the data, which belongs to one cluster, should be as similar as possible.

2- Heterogeneity between the clusters: the data, which belongs to different clusters, should be as different as possible.

The membership functions do not reflect the actual data distribution in the input and the output spaces. They may not be suitable for fuzzy pattern recognition. To build membership functions from the data available, a clustering technique may be used to partition the data, and then produce membership functions from the resulting clustering.

"Clustering" is a process to obtain a partition $P$ of a set $E$ of $N$ objects $x_{i}(i=1,2, \ldots, N)$, using the resemblance or disemblance measure, such as a distance measure $d$. A partition $P$ is a set of disjoint subsets of $E$ and the element $P s$ of $P$ is called cluster and the centers of the clusters are called centroids or prototypes. Many techniques have been developed for clustering data. In this paper c-means clustering is used. It's a simple unsupervised learning method which can be used for data grouping or classification when the number of the clusters is known. It consists of the following steps:

Step 1:

Choose the number of clusters $-K$

Step 2:

Set initial centers of clusters $c_{1}, c_{2}, \ldots, c_{k}$;

Step 3:

Classify each vector

$x_{1}=\left[x_{11}, x_{12}, \ldots . x_{1 n}\right]^{\top}$ into the closest center $c_{i}$ by Euclidean distance measure: 
$\left\|x_{i}-c_{i}\right\|=\min \left\|x_{i}-c_{i}\right\|$

Step 4:

Recompute the estimates for the cluster centers $c_{i}$ let $c_{i}=\left[c_{i 1}, c_{i 2}, \ldots . . c_{i n}\right]^{\top}$

$C_{\text {im }}$ be computed by:

$c_{i m}=\frac{\sum x_{l i} \in \operatorname{cluster}\left(i^{x \lim }\right)}{N_{i}}$

Where $N_{i}$ is the number of vectors in the i-th cluster.

Step 5:

If none of the cluster centers ( $c i=1,2, \ldots, k$ ) changes in step4

Stop; otherwise go to step 3.

\section{2. 1 C-means algorithm}

The criterion function used for the clustering process is:

$$
J(v)=\sum_{i=1}^{c} \sum_{k=1}^{n} u_{i k}^{m}\left|x_{k}-v_{i}\right|^{2},
$$

Where:

$x_{1, \ldots} x_{n}-' n$ ' data sample vectors;

$v_{1, \ldots,}, v_{c} \quad$-'c' denotes cluster centrers (centroids);

$u=u_{i k}$ matrix, where $u_{i k} c x m$ is the $i$-th membership value of the $k$-th input sample $x_{k}$, and the membership values satisfy the following conditions:

$0 \leq u_{i k} \leq 1 ; i=1, \ldots, c ; k=1, \ldots, n$;

$\sum_{i=1}^{c} u_{i k}=1 ; k=1, \ldots, n ; k=1, \ldots, n$;

$0<\sum_{k=1}^{n} u_{i k}\langle 1 ; i=1, \ldots, c$;

$m \in[1, \infty)$ is an exponent weight factor.

The Fuzzy Logic Toolbox command line function, fcm, starts with an initial guess for the cluster centers, which are intended to mark the mean location of each cluster. The initial guess for these cluster centers is most likely incorrect. Next, fcm assigns every data point a membership grade for each cluster. By iteratively updating the cluster centers and the membership grades for each data point, fcm iteratively moves the cluster centers to the right location within a data set.

\subsection{Local Intensities Weighting (LIW)}

Otsu method assumed that the criterion function as in Eq. (7) was always unimodal, but as shown by Kittler and Illingworth [19] that this assumption is not always hold in general. They showed some class of image statistics: unimodal, bimodal, trimodal, or multimodal and the Otsu criterion function for this kind of images is not always unimodal. They proposed another criterions supposing that the criterion function of Otsu method of an image is not unimodal. It was also stated that the unimodal histogram will be characterized by a unimodal objective function of Otsu method.

In thresholding the image, the Otsu method used the histogram of image. The criterion function is derived from this histogram. Rather than proposing a new criterion to improve Otsu method, this paper takes on a different strategy. The idea is to make the thermal image always has the unimodal histogram. To achieve this, a local neighborhood operation (which we call LIW) is applied to the thermal image before passing it to the Otsu method for detection of the defect. 
This technique will brighten the bright area and darken the dark area. It is assumed that the defect is represented by the brighter area, and the background or sound area is the darker area [20]. Therefore, the defective pixels will be shifted to 255 and non-defective pixels tend to 0 values.

Consider a pixel $f(i, j)$ in a thermal image with its 8-connectivity configuration as shown in Fig.2, where: $z 1=f(i-1, j-1), z 2=f(i-1, j), z 3=f(i-1, j+1), z 4=f(i, j-1), z 5=f(i, j), z 6=f(i, j+1), z 7=f(i+1, j-1)$,

$z 8=f(i+1, j)$

And $z 9=f(i+1, j+1)$.

\begin{tabular}{|l|l|l|}
\hline$z 1$ & $z 2$ & $z 3$ \\
\hline$z 4$ & $z 5$ & $z 6$ \\
\hline$z 7$ & $z 8$ & $z 9$ \\
\hline
\end{tabular}

Fig.1. Liw window

Weighted intensities pixel $g(i, j)$ for a new image is defined as a multiplication of the pixels reside in the local window as defined in Eq. (8). Fig.3 illustrates the weighting process. This is done through out the entire image as a convolution process. These values are then normalized by dividing with the maximum value.

$g(i, j)=z 1^{\star} z 2^{\star} z 3^{\star} z 4^{\star} z 5^{\star} z 6^{\star} z 7^{\star} z 8^{\star} z 9$

(8)

$z 1^{\star} z 2^{\star} z 3^{\star} z 4^{\star} z 5^{\star} z 6^{\star} z 7^{\star} z 8^{\star} z 9$
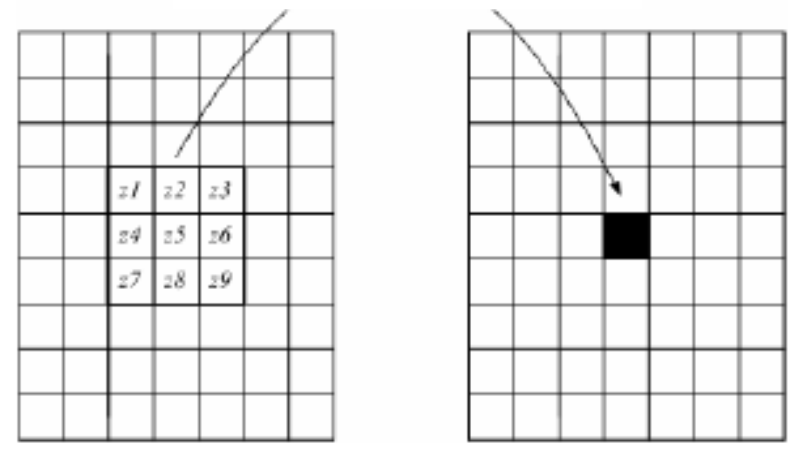

Fig. 2. Liw operation

\section{3- Thermographic measurements}

Thermography is a convenient, non contact method for making temperature field measurements [21]. The data of the temperature field can be used to obtain information on thermal phenomena which occur during the working process. The vision system elaborated within the framework of the research described in this work has been assigned to steel and iron process. The system has included hardware and software parts. The hardware part has consisted of a camera and a portable PC to make recordings in real time. The main task of this part was to observe the process by means of IR camera. The device used was a FLIR ThermaCAM A40 imaging system. It has a $240 \times 320$ pixels focal-plane-array uncooled microbolometer detector, with a sensitive range of $7.5-13 \mathrm{~mm}$. Imaging and storage was made at a frequency rate of $50 \mathrm{~Hz}$. The PC is equipped by powerful software that is ThermaCam Researcher 2.9, this software is used to analyse dynamic IR radiation records including the emissivity calculations.

Table 1. Specification of thermo-cam

\begin{tabular}{ll}
\hline Thermo-cam & - Solide state, uncooled micro bolometer detector, \\
& 7.5 to $13 \mu \mathrm{m}$ \\
(FLIR-A 40 series) & $--40^{\circ} \mathrm{C}$ to $+2000^{\circ} \mathrm{C}$ storage temperature range \\
& - Solide object materials and emissivity: 0.1 to 0.95 \\
& - For short distance, humidity is default value of $50 \%$ \\
& $-0.08^{\circ} \mathrm{C}$ at $30^{\circ} \mathrm{C}$ thermal sensitivity
\end{tabular}




\section{Results and discussion}

With using infrared camera and appropriate software used for analysis of thermal images, we have possibility to detect in concrete installation area possessing highest temperature and measure the temperature in real-time. In this section we will present the result's obtained using thresholding methods. Indeed, the inspection made on the level of Iron and steel plants using an infrared camera allows the visualisation of some defects existing on the latter.

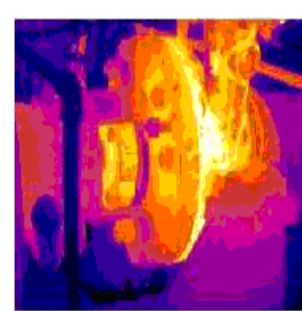

(a)

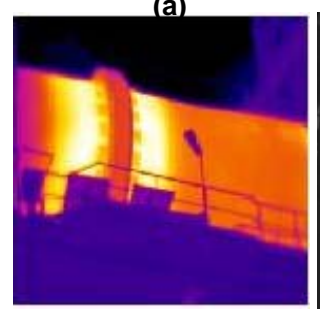

(a)

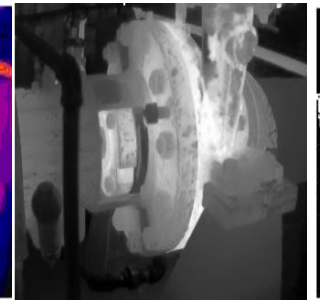

(b)

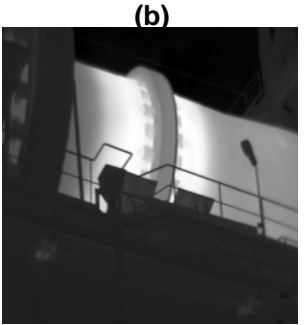

(b)

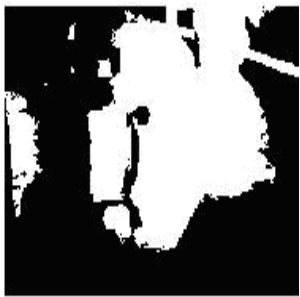

(c)

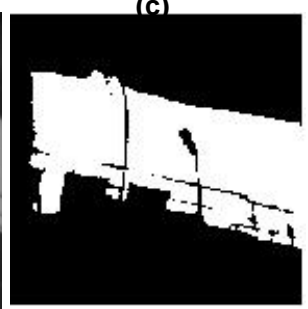

(c)

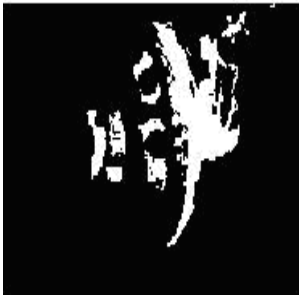

(d)

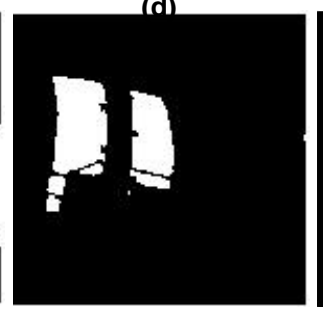

(d)

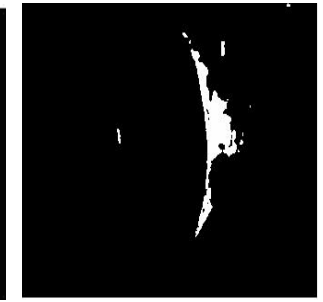

(e)

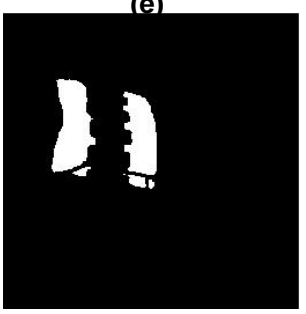

(e)

Fig. 3. Thresholding results of iron and steel industry images: (a) original, (b) gray image, (c) Otsu's method, (d) Fuzzy cmeans's method, (e) LIW's method

\section{Conclusion}

Infrared thermography proved very useful for the detection and determination of defects in Iron and Steel industry, but because of the complexity of the structure and the performance of the machines, many precautions must be taken during the input and interpretation of thermographic data. In this study, we have investigated the effectiveness of three thresholding methods through infrared thermal images of Iron and Steel plant. To evaluate the quality of these thresholding techniques, we have made a comparison between the resulted binary images and we have seen that LIW method give a good detection of interest zone (defect zone) than Fuzzy c-means and Otsu methods. The proposed approach allows a non-destructive inspection, it improves the Iron and steel process maintenance and gives a global quality evaluation and reduces the quality control cost. Since there is only a single infrared camera needed which makes a fast evaluation possible.

\section{REFERENCES}

[1] M.E. Yuksel, "Edge detection in noisy images by neuro-fuzzy processing". AEU Int J Electron Commun 2007;61:82-9.

[2] S.H. Kwon, "Threshold selection based on cluster analysis", Pattern Recognition Lett 2004; 25:1045-50.

[3] S. Wang, F. Chung, F. Xiong, "A novel image thresholding method based on parzen window estimate", Pattern Recognition, 2008; 41:117-29.

[4] R. Demirci, Rule-based automatic segmentation of color images,AE U Int J Electron Commun, 2006; 60:435-42.

[5] T. Sund, K. Eilertsen, "An algorithm for fast adaptive binarization with applications in radiotherapy imaging", IEEE Trans Med Imaging, 2003; 22:22-8.

[6] Y. Solihin, C.G. Leedham, "Integral ratio: a new class of global thresholding techniques for handwriting images", IEEE Trans Pattern Anal Mach Intell, 1999; 21:761-8.

[7] B. Bhanu," Automatic target recognition: state of the art survey, IEEE Trans Aerosp Electron Syst 1986; 22:364-79.

[8] L. Bruzzone, D.F. Prieto. Automatic analysis of the difference image for unsupervised change detection, IEEE Trans Geosci Remote Sensing 2000; 38:1171-82.

[9] W.H. Tsai, "Moment-preserving thresholding: a new approach”, Comput Vis Graph Image Process, 1985; 29:377-93. 
[10] Y. Bazi, L. Bruzzone, F. Melgani, "Image thresholding based on the EM algorithm and the generalized Gaussian distribution, Pattern Recognition, 2007; 40:619-34.

[11] N.Otsu, "A threshold selection method from gray-level histograms", IEEE Trans Syst Man Cybern, 1979; 9:62-6.

[12] P.K. Sahoo, S. Soltani, A.K.C. Wong, "A survey of thresholding techniques", Comput Vis Graph Image Process ,1988; 41:233-60.

[13] Z. Hou, Q. Hu, W.L . Nowinski, “On minimum variance thresholding”, Pattern Recognition Lett, 2006; 27:1732-43.

[14] T. Pun, "A new method for grey-level picture thresholding using the entropy of the histogram", Signal Process ,1980;2:223-37.

[15] J.N. Kapur, P.K. Sahoo, A.K.C. Wong. A new method for grey-level picture thresholding using the entropy of the histogram", Comput Vis Graph Image Process, 1985; 29:273-85.

[16] D. Liu, Z.H. Jiang, H.Q. Feng, "A novel fuzzy classification entropy approach to image thresholding", Pattern Recognition Lett, 2006; 27:1968-75.

[17] P.K. Saha, J.K. Udupa, "Optimum image thresholding via class uncertainty and region homogeneity", IEEE Trans Pattern Anal Mach Intell, 2001; 23:689-706.

[18] R. Krasteva, "Bulgarian Hand-Printed Character Recognition Using Fuzzy C-Means Clustering", Bulgarian Academy of sciences problems of engineering cybernetics and robotics, 53, pp. 112-117.

[19] J. Kittler, J. Illingworth, "On Threshold Selection using Clustering Criteria", IEEE Trans. on Systems, Man \& Cybernetics, Vol. SMC-15, No. 5, 1985, pp. 652-655.

[20] R. Heriansyah, S. A. R. Abu-Bakar, "Defect Detection in Thermal Image using Thresholding Technique" 6th WSEAS International Conference on CIRCUITS, SYSTEMS, ELECTRONICS,CONTROL \& SIGNAL PROCESSING, Cairo, Egypt, 2007,Dec 29-31.

[21] S. Matteï, D. Grevey, A. Mathieu, L. Kirchner, "Using infrared thermography in order to compare laser and hybrid (laser + MIG) welding processes", Optics \& Laser Technology, 41, 2009, pp. 665-670. 